\title{
Relaciones longitud-biomasa en macroinvertebrados bentónicos de un arroyo serrano del sur de Córdoba, Argentina
}

\author{
Cristina Gualdoni ${ }^{\varpi}$; Patricio W. French \& Ana M. Oberto
}

Dpto. de Ciencias Naturales, Facultad de Ciencias Exactas, Físico-Químicas y Naturales, Universidad Nacional de Río Cuarto. Río Cuarto, Córdoba, Argentina

\begin{abstract}
Resumen. Los métodos más utilizados para la determinación indirecta de la biomasa de invertebrados se basan en ecuaciones matemáticas que relacionan dimensiones lineales del cuerpo y el peso del organismo. Sin embargo, estas relaciones presentan variaciones que dependen de las condiciones bióticas y fisicoquímicas de cada ambiente particular. El propósito de este estudio fue determinar las ecuaciones que permitan estimar la relación entre la talla y el peso de macroinvertebrados bentónicos del arroyo Achiras, situado en el sudoeste de la provincia de Córdoba (Argentina). El bentos se colectó mediante redes de Surber, con una frecuencia estacional (otoño, invierno, primavera y verano) entre 2006 y 2007. Se midió la longitud corporal y se registró el peso seco de organismos de los grupos taxonómicos más representados y se calcularon las ecuaciones de regresión que mejor explicaran la relación entre ambas variables. Se evaluaron tres modelos, lineal, potencial y exponencial, y se seleccionó la expresión que asociara mejor las variables. Se estimaron ecuaciones para 24 taxones de macroinvertebrados (tres especies, 12 géneros, tres subfamilias, cuatro familias y dos órdenes). La mayoría de las regresiones fueron altamente significativas y explicaron una gran proporción de la variación del peso seco. Las ecuaciones estimadas sugieren que para la mayoría de los macroinvertebrados bentónicos del arroyo Achiras, la relación entre talla y biomasa es alométrica y puede ser explicada por un modelo potencial o exponencial, mientras que, solo para cuatro taxones la regresión lineal simple ajustó mejor los valores de longitud y peso corporal. Los modelos de regresión aplicados a grupos con menor nivel taxonómico (familia y género) explicaron una mayor proporción de la variación del peso, respecto a las regresiones al nivel de orden. Además se estimaron dos ecuaciones generales que se ajustaron al modelo potencial. En la mayoría de los grupos taxonómicos analizados el valor de la pendiente fue menor a tres y el coeficiente de determinación promedio fue de 0.77 . Ambos parámetros se ubican dentro del rango de variación de los resultados publicados para la fauna bentónica de otros ambientes lóticos.
\end{abstract}

[Palabras clave: macroinvertebrados de agua dulce, regresiones longitud-biomasa, arroyos, relaciones biométricas]

\begin{abstract}
AвSTRACT. Length-biomass relationships in benthic macroinvertebrates of a mountain stream of the south of Córdoba, Argentina: The methods used for indirect determination of invertebrates biomass are based on mathematical equations that relate body linear dimensions and weight of the organism. However, these relationships depend on biotic and physicochemical conditions of each particular environment. The aim of this study was to determine the equations to estimate the relationship between lenght and weight of benthic macroinvertebrate of Achiras stream, located in the southwest of Córdoba province (Argentina). The benthos was collected with Surber nets, with a seasonal occurrence (fall, winter, spring and summer), between 2006 and 2007. Body length and dry weight for the specimens of the more represented taxonomic groups were registered and the regression equations that best explain the relationship between the two variables were calculated. We evaluated three models, simple linear, potential and exponential, and we selected the better expression that associate the variables. Equations were estimated for 24 macroinvertebrates taxa (three species, 12 genera, three subfamilies, four families and two orders). Most regressions were highly significant and explain a large proportion of the variation of the dry weight. The estimated equations suggest that for most of the benthic macroinvertebrates of Achiras stream, the relationship between length and biomass is alometric and can be explained by a power or exponential model, while only for four taxa simple linear regression best fit length and body weight values. Regression models applied to lower level taxonomic groups (family and genus) explained a greater proportion of the variation in weight, with regard to the order level regressions. In addition two general equations were estimated following the power model. In most analyzed taxa the value of the slope was less than three, and the average determination coefficient found in our study was 0.77 , which coincide with the variation range of the published results for benthic fauna of other lotic environments.
\end{abstract}

[Keywords: freshwater macroinvertebrates, length-biomass regressions, streams, biometric relationships]

\section{INTRODUCCIÓN}

Uno de los aspectos más importantes para comprender la ecología de las poblaciones y su regulación en los ecosistemas lóticos es

Editor asociado: Fernando Unrein

$\triangle$ cgualdoni@exa.unrc.edu.ar la valoración de la producción secundaria y la transferencia espacial de energía dentro y entre los hábitats, y a través del continuo fluvial. El punto de partida para arribar a 
estas evaluaciones es el conocimiento de la biomasa que circula en el ecosistema. $\mathrm{Si}$ bien esta variable se puede determinar de forma directa pesando los organismos, las estimaciones de biomasa de invertebrados a partir de ecuaciones matemáticas que relacionan la talla y el peso se cuentan entre las alternativas más aceptadas ya que son más rápidas y precisas que otros métodos (Benke et al. 1999). Aunque es deseable contar con ecuaciones precisas para cada grupo taxonómico y cada región en particular, con el fin de economizar tiempo y recursos, a menudo se obtienen determinaciones de biomasa a partir de ecuaciones de regresión longitud-biomasa ya publicadas (Benke 1996). Se han desarrollado ecuaciones predictivas para invertebrados de agua dulce de Europa (Meyer 1989; Burgherr \& Meyer 1997; González 2002), Nueva Zelanda (Stoffels et al. 2003; Towers et al. 1994), América del Norte (Smock 1980; Benke et al. 1999; Johnston \& Cynjak 1999), América del Sur (Cressa 1986, 1999a,b) y para Argentina (Miserendino 2001). Aún cuando se consideren poblaciones de una misma especie, las colecciones provenientes de distintos lugares, presentarán variaciones en las relaciones de longitud-biomasa causadas por diferencias en las condiciones de cada ambiente. Tanto la talla como el peso de los organismos dependen de la disponibilidad de alimento, de las características físico-químicas del hábitat y de las condiciones genéticas o del estatus trófico de la especie. Por lo tanto es recomendable que para las determinaciones de biomasa se utilicen ecuaciones ajustadas para cada región en particular.

Enla mayoría delos estudios se suponequelos animales presentan un crecimiento alométrico y se determina la biomasa como una función de la longitud corporal siguiendo un modelo potencial. En dicho modelo, el exponente "b" (la pendiente de la regresión lineal) tiene un valor cercano a tres, lo cual representa una relación cúbica perfecta entre la longitud y el peso de los organismos (Benke et al. 1999). Sin embargo, el crecimiento alométrico también brinda la posibilidad de que las variables de longitud y biomasa se ajusten mejor a través de un modelo exponencial. Además, en algunos organismos ambas variables se incrementan de manera semejante, lo que implica un crecimiento isométrico $\mathrm{y}$, por lo tanto, el modelo de regresión más apropiado es el lineal simple. Aunque en menor proporción, estos dos últimos modelos han sido aplicados a la relación talla-biomasa en organismos del bentos de agua dulce (Cressa 1986, 1999a,b;
Meyer 1989; Burgeherr \& Meyer 1997; Benke et al. 1999; Johnston \& Cunjak 1999; González et al. 2002; Becker et al. 2009).

La regulación de ríos y arroyos mediante presas se suma a las presiones que desde hace décadas afectan los ecosistemas fluviales. La instauración de una presa reestructura las comunidades a lo largo del continuo e impone nuevas condiciones ambientales que influyen en la abundancia y composición de la biota, en su tasa de desarrollo y en los niveles de productividad (Armitage 2006; Poff \& Zimmerman 2010). En la cuenca alta sistema Achiras-del Gato, que drena el sur de las Sierras de los Comechingones (Córdoba, Argentina), recientemente finalizó la construcción de una presa de mediana envergadura. Estudios previos a la regulación del arroyo han aportado datos sobre la estructura y la diversidad de la comunidad bentónica y de la fracción derivante (Gualdoni \& Oberto 2012). La estimación de su biomasa a partir de muestras colectadas antes de la regulación representa una herramienta valiosa para monitorear las variaciones de producción secundaria que resulten de las nuevas condiciones hidráulicas y fisicoquímicas río abajo de la presa. El propósito de este estudio es determinar las ecuaciones que permitan estimar la relación entre longitud y biomasa corporal de los principales grupos taxonómicos del macrobentos del arroyo Achiras (Córdoba, Argentina).

\section{Materiales y Métodos}

Los macroinvertebrados bentónicos se colectaron en dos tramos pedemontanos del arroyo Achiras, en la falda sudoriental de las Sierras de los Comechingones, entre 810 y 753 m.s.n.m., y entre los

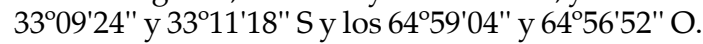
$\mathrm{El}$ arroyo pertenece al sistema endorreico Achirasdel Gato, ubicado en el sudoeste de la Provincia de Córdoba (Argentina). Se trata de un curso de agua deflujo permanente que depende del aporte freático, con variación de caudal debido a las precipitaciones y picos de creciente en la época estival. En la zona de estudio, donde el arroyo es de orden 6, la corriente fluye en una sucesión longitudinal de rabiones con turbulencia y velocidades de corriente más elevadas y substrato de roca y bloque, correderas con moderada velocidad de corriente, turbulencia escasa o ausente y sustrato de gravas y arenas, y pozones más profundos con fondo de arena y limo. Sus aguas son bicarbonatadas cálcicas, y como la mayoría de los arroyos serranos de la región, poseen buena calidad y están saturadas de oxígeno. Más detalles sobre las características físicas, químicas y biológicas del arroyo y su cuenca de drenaje pueden consultarse en Gualdoni \& Oberto (2012). 
Los muestreos se realizaron mediante redes de Surber de $0.09 \mathrm{~m}^{2}$ de área y $300 \mu \mathrm{m}$ de abertura de malla, en hábitats de correderas y rabiones, durante las cuatro estaciones del año, entre 2006 y 2007. Las muestras se fijaron en el campo con formaldehído al $4 \%$ y en el laboratorio los organismos se lavaron con agua y se separaron bajo estereomicroscopio y microscopio binocular. La totalidad de las muestras fue procesada en un plazo no mayor a 12 meses. En coincidencia con Stoffels et al. (2003), nosotros asumimos que la pérdida de peso debido a la preservación fue mínima. Las determinaciones taxonómicas se realizaron con la máxima resolución posible, según el conocimiento de la fauna argentina (Lopreto \& Tell 1995; Fernández \& Domínguez 2001; Domínguez \& Fernández 2009).

Se investigó la relación entre la talla (longitud) y biomasa (peso seco) en los taxones más abundantes y frecuentes de la fauna bentónica colectada. Las mediciones se realizaron con oculares micrométricos de 4 y 10 aumentos (error de $0.1 \mathrm{~mm}$ ), montado en una lupa estereoscópica. En los artrópodos se midió la longitud corporal (LC) como la distancia entre el borde anterior de la cápsula cefálica y el borde posterior del último segmento abdominal. En los moluscos gasterópodos LC se midió como el eje mayor de la concha (Meyer 1989). Luego de medidos, los organismos se transfirieron a placas de aluminioindividuales, de peso conocido y se secaron en estufa a $60^{\circ} \mathrm{C}$ durante $48 \mathrm{~h}$. El peso seco (P) se registró mediante una balanza electrónica (OHAVS Analytical Plus, AP 250D-0OHAVS Analytical Plus, AP 250D-0) con precisión de $0.01 \mathrm{mg}$. Cuando los especímenes fueron muy pequeños o muy livianos, varios organismos (hasta ocho por placa) fueron secados y pesados juntos. Los tricópteros se pesaron sin estuches y los gasterópodos se secaron y pesaron con sus conchas intactas. Las dimensiones lineales fueron registradas en milímetros y el peso seco en miligramos.

Las relaciones entre longitud y biomasa se estimaron para todas las especies, géneros y subfamilias en los que se pudieron medir al menos 10 ejemplares. Las relaciones a nivel familia y orden se desarrollaron agrupando los datos individuales de los organismos correspondientes a cada familia y a cada orden. Del mismo modo se estimaron dos ecuaciones generales representativas de los grandes grupos faunísticos del arroyo Achiras: para la totalidad de los Insecta y para todos los macroinvertebrados bentónicos medidos. Los valores pareados de talla y peso seco de cada grupo taxonómico se procesaron, mediante el programa estadístico InfoStat/Profesional Versión 1.1 (Di Rienzo et al. 2010). Para estimar la ecuación de regresión que mejor explicara las relaciones entre LC y $\mathrm{P}$, se evaluaron 3 modelos de regresión: el modelo lineal $\left(\mathrm{P}=\mathrm{a}+\mathrm{b}^{*} \mathrm{LC}\right)$, el potencial $\left(\mathrm{P}=\mathrm{a}^{*} \mathrm{~L} \mathrm{C}^{\mathrm{b}}\right.$ ó su equivalente $\left.\ln \mathrm{P}=\ln \mathrm{a}+\ln \mathrm{LC}^{*} \mathrm{~b}\right)$ y el exponencial $(\ln \mathrm{P}=\ln \mathrm{a}+\mathrm{LC} * \mathrm{~b})$, donde $\mathrm{P}=$ peso seco; $\mathrm{LC}=$ longitud corporal; $\mathrm{a}=$ ordenada al origen; $\mathrm{b}=$ pendiente. Para seleccionar la expresión que asociara mejor a las variables de longitud y biomasa se consideró el mayor valor del coeficiente de correlación $\mathrm{R}^{2}$ y el menor cuadrado medio (CM) del error respecto del CM de la variable independiente. Los supuestos se comprobaron mediante diagrama de dispersión (homogeneidad de varianza), de residuos versus valores predichos para el peso y "Q-Q Plot" (distribución normal de los datos versus residuos). Para ajustar las ecuaciones, se eliminaron los datos indicados como "outliers". Para conocer si era necesaria la transformación de los datos, se realizó la Prueba de Atkinson.

\section{RESULTADOS}

Del total de la fauna bentónica colectada se seleccionaron los 18 taxones más numerosos $y$ frecuentes (85\% de la abundancia total estimada) para medir y pesar los organismos individualmente (Tabla 1). Los taxones escogidos pertenecen a diferentes niveles de resolución taxonómica (especies, géneros y subfamilias). Las variables se registraron en 667 macroinvertebrados, sin embargo, debido a que en el análisis de cada grupo se eliminaron los puntos que resultaron "outliers", las ecuaciones se ajustaron sobre un total de 633 individuos.

Tabla 1. Taxones bentónicos del arroyo Achiras incluidos en el análisis. A: adulto; L: larva; N: ninfa. *: niveles taxonómicos en los cuales las ecuaciones se estimaron a partir de la agrupación de datos de los grupos de menor nivel taxonómico.

Table 1. Benthic taxa of Achiras stream included in the analysis. A: adult; L: larva; N: nymph *: taxonomic levels in which equations were estimated from the summation of data from groups of lower taxonomic level.

\begin{tabular}{|c|c|c|}
\hline \multicolumn{2}{|l|}{ Taxón } & \multirow[t]{2}{*}{ Estado } \\
\hline Gasteropoda & Lymnaea sp. & \\
\hline \multirow[t]{6}{*}{ Ephemeroptera * } & Baetidae * & $\mathrm{N}$ \\
\hline & Americabaetis sp. & $\mathrm{N}$ \\
\hline & Camelobaetidius penai & $\mathrm{N}$ \\
\hline & Leptohyphidae * & $\mathrm{N}$ \\
\hline & Leptohypes eximius & $\mathrm{N}$ \\
\hline & $\begin{array}{l}\text { Trichorythodes } \\
\text { popayanicus }\end{array}$ & $\mathrm{N}$ \\
\hline Odonata & Progomphus sp. & $\mathrm{N}$ \\
\hline Trichoptera & Smicridea sp. & $\mathrm{L}$ \\
\hline Lepidoptera & Petrophila sp. & $\mathrm{L}$ \\
\hline \multirow[t]{7}{*}{ Coleoptera } & Elmidae * & A y L \\
\hline & Austrelmis sp. & A y L \\
\hline & Heterelmis sp. & A y L \\
\hline & Macrelmis sp. & A y L \\
\hline & Cylloepus sp. & A \\
\hline & Microcylloepus sp. & A \\
\hline & Helichus sp. & A \\
\hline \multirow[t]{5}{*}{ Diptera * } & Chironomidae * & $\mathrm{L}$ \\
\hline & Chironominae & $\mathrm{L}$ \\
\hline & Tanypodinae & $\mathrm{L}$ \\
\hline & Orthocladinae & $\mathrm{L}$ \\
\hline & Simulium sp. & L y P \\
\hline
\end{tabular}


Tabla 2. Parámetros de la regresión lineal (ln $\left.\mathrm{P}=\ln \mathrm{a}+\mathrm{b}^{*} \ln \mathrm{LC}\right)$ según el modelo potencial para la relación entre la longitud corporal (LC) y peso seco (P) de algunos macroinvertebrados analizados. a y b: constantes de la regresión; $\mathrm{R}^{2}$ : coeficiente de determinación; XL: longitud promedio; RL: rango de los valores de la variable independiente; XP: peso promedio; RP: rango de los valores de la variable dependiente; $n$ : numero de especímenes.

Table 2. Parameters of the linear regression $\left(\ln \mathrm{P}=\ln \mathrm{a}+\mathrm{b}^{*} \ln \mathrm{LC}\right)$ according to power model for the relation between body length (LC) and dry weight (P) of some macroinvertebrates analyzed. $a$ and $b$ : regression constants; $\mathrm{R}^{2}$ : determination coefficient; XL: average length; RL: values range of the independent variable; XP: average weight; RP: values range of the dependent variable; $n$ : specimens number.

\begin{tabular}{|c|c|c|c|c|c|c|c|c|}
\hline Taxón & $\ln \mathrm{a}$ & $\mathrm{b}$ & $\mathrm{R}^{2}$ & $\mathrm{XL}$ & RL & $\mathrm{XP}$ & $\mathrm{RP}$ & $\mathrm{n}$ \\
\hline \multicolumn{9}{|l|}{ Gasteropoda } \\
\hline Lymnaea sp. & $-3.05 \pm 0.09$ & $2.60 \pm 0.07$ & 0.98 & 3.24 & $1.2-8.2$ & 1,449 & $0.107-13.640$ & 31 \\
\hline \multicolumn{9}{|l|}{ Ephemeroptera } \\
\hline $\begin{array}{l}\text { Americabaetis sp. } \\
\text { Odonata }\end{array}$ & $-4.73 \pm 0.10$ & $2.21 \pm 0.08$ & 0.96 & 3.69 & $1.0-7.8$ & \multicolumn{2}{|c|}{ Odonata } & 32 \\
\hline $\begin{array}{l}\text { Progomphus sp. } \\
\text { Lepidoptera }\end{array}$ & $-4.20 \pm 0.20$ & $2.41 \pm 0.10$ & 0.99 & 8.89 & $1.5-19.9$ & 6.156 & $0.055-25.960$ & 11 \\
\hline $\begin{array}{l}\text { Petrophila sp. } \\
\text { Coleoptera }\end{array}$ & $-5.04 \pm 0.35$ & $2.38 \pm 0.14$ & 0.94 & 12.15 & $3.2-22.9$ & 3.800 & $0.070-15.520$ & 19 \\
\hline Austrelmis sp. (L) & $-5.40 \pm 0.21$ & $2.62 \pm 0.14$ & 0.90 & 4.28 & $1.9-7.6$ & 0.271 & $0.007-0.920$ & 40 \\
\hline Heterelmis sp. (L) & $-4.38 \pm 0.36$ & $1.62 \pm 0.24$ & 0.66 & 4.35 & $2.1-6.6$ & 0.158 & $0.025-0.390$ & 25 \\
\hline Macrelmis sp. (L) & $-6.72 \pm 0.79$ & $3.14 \pm 0.42$ & 0.75 & 6.43 & $3.4-10.5$ & 0.57 & $0.020-1.720$ & 21 \\
\hline Austrelmis sp. (A) & $-3.17 \pm 0.64$ & $2.39 \pm 0.50$ & 0.65 & 3.54 & $2.8-3.9$ & 0.875 & $0.390-1.390$ & 14 \\
\hline Heterelmis sp. (A) & $-3.18 \pm 0.65$ & $2.32 \pm 0.66$ & 0.38 & 2.72 & $2.4-3.2$ & 0.443 & $0.270-0.920$ & 22 \\
\hline Macrelmis sp. (A) & $-1.82 \pm 0.96$ & $1.40 \pm 0.61$ & 0.36 & 4.69 & $4.3-5.1$ & 1,415 & $1.090-1.740$ & 11 \\
\hline Helichus sp. (A) & $-2.45 \pm 0.41$ & $2.12 \pm 0.23$ & 0.91 & 6.04 & $5.5-7.0$ & 3.909 & $3.200-5.840$ & 10 \\
\hline \multicolumn{9}{|l|}{ Diptera } \\
\hline Tanypodinae & $-4.65 \pm 0.20$ & $1.80 \pm 0.12$ & 0.89 & 5.48 & $1.8-9.5$ & 0.248 & $0.025-0.670$ & 30 \\
\hline Orthocladinae & $-3.90 \pm 0.21$ & $1.27 \pm 0.15$ & 0.70 & 3.98 & $1.6-8.0$ & 0.128 & $0.020-0.350$ & 33 \\
\hline Simulium sp. (L) & $-3.31 \pm 0.18$ & $1.29 \pm 0.14$ & 0.79 & 3.42 & $1.6-5.7$ & 0.187 & $0.063-0.430$ & 24 \\
\hline Simulium sp. $(\mathrm{P})$ & $-1.72 \pm 0.32$ & $0.52 \pm 0.34$ & 0.20 & 2.55 & $1.9-3.0$ & 0.300 & $0.180-0.460$ & 11 \\
\hline
\end{tabular}

Las longitudes corporales medidas estuvieron dentro del rango $0.7-22.9 \mathrm{~mm}$ y los pesos entre 0.007 y $25.9 \mathrm{mg}$. Las regresiones obtenidasfueron altamente significativas para la mayoría de los grupos $(0.0001<P<0.001)$, y explicaron una gran proporción de la variación del peso seco $\left(\mathrm{R}^{2}=0.66-0.99\right)$. No fue posible obtener modelos muy apropiados para los organismos adultos de algunos géneros de Elmidae $(0.0005<P<0.11$ y $\left.R^{2}=0.36-0.65\right)$ ni para las pupas de Simulium sp. $\left(P=0.37\right.$ y $\left.\mathrm{R}^{2}=0.20\right)$.
Para la mayoría de los niveles taxonómicos de especie, género y subfamilia el modelo potencial fue el que mejor ajustó los datos de las variables LC y P (Tabla 2). En Lymnaea sp., Americabaetis sp., Progomphus sp., Petrophila sp., larvas de Austrelmis sp. y Helichus sp. se obtuvieron valores de $\mathrm{R}^{2}$ iguales o superiores a 0.90 , mientras que en adultos de Heterelmis sp. y Macrelmis sp. y en pupas de Simulium sp., fue bajo.

Tabla 3. Parámetros de la regresión lineal ( $\ln \mathrm{P}=\ln \mathrm{a}+\mathrm{b}^{*} \mathrm{LC}$ ) según el modelo exponencial para la relación entre la longitud corporal (LC) y peso seco (P) de algunos macroinvertebrados analizados. Abreviaturas en leyenda de Tabla 2.

Table 3. Parameters of the linear regression $\left(\ln \mathrm{P}=\ln \mathrm{a}+\mathrm{b}^{*} \mathrm{LC}\right)$ according to exponential model for the relation between body length (LC) and dry weight (P) of some macroinvertebrates analyzed. Abbreviations in legend of Table 2.

\begin{tabular}{lcccccccc}
\hline Taxón & $\ln \mathrm{a}$ & $\mathrm{b}$ & $\mathrm{R}^{2}$ & $\mathrm{XL}$ & $\mathrm{RL}$ & $\mathrm{XP}$ & $\mathrm{RP}$ & $\mathrm{n}$ \\
\hline Ephemeroptera & & & & & & & & \\
$\quad$ L. eximius & $-2.99 \pm 0.10$ & $0.52 \pm 0.02$ & 0.94 & 3.82 & $0.7-6.6$ & 0.525 & $0.075-1.650$ & 33 \\
$\quad \begin{array}{l}\text { T. popayanicus } \\
\text { Trichoptera }\end{array}$ & $-3.00 \pm 0.09$ & $0.53 \pm 0.03$ & 0.90 & 2.63 & $1.0-5.5$ & 0.253 & $0.045-0.970$ & 32 \\
$\quad$ & & & & & & & & \\
Smicridea sp. & $-3.46 \pm 0.17$ & $0.44 \pm 0.02$ & 0.96 & 6.57 & $2.7-11.1$ & 1.002 & $0.095-3.320$ & 19 \\
$\quad$ Coleoptera & & & & & & & & \\
Heterelmis sp. (A) & $-3.25 \pm 0.66$ & $0.88 \pm 0.25$ & 0.39 & 2.72 & $2.4-3.2$ & 0.443 & $0.270-0.920$ & 11 \\
Macrelmis sp. (A) & $-1.06 \pm 0.62$ & $0.30 \pm 0.13$ & 0.36 & 4.69 & $4.3-5.1$ & 1.415 & $1.090-1.740$ & 15 \\
Helichus sp. (A) & $-0.76 \pm 0.22$ & $0.35 \pm 0.04$ & 0.92 & 6.04 & $5.5-7.0$ & 3.909 & $3.200-5.840$ & 10 \\
$\quad$ Diptera & & & & & & & & \\
$\quad$ Chironominae & $-3.52 \pm 0.14$ & $0.33 \pm 0.02$ & 0.85 & 5.36 & $2.2-9.6$ & 0.215 & $0.045-0.810$ & 32 \\
$\quad$ Tanypodinae & $-3.84 \pm 0.14$ & $0.37 \pm 0.02$ & 0.90 & 5.48 & $1.8-9.5$ & 0.248 & $0.025-0.670$ & 30 \\
Simulium sp. (L) & $-3.21 \pm 0.15$ & $0.41 \pm 0.04$ & 0.80 & 3.42 & $1.6-5.7$ & 0.187 & $0.063-0.430$ & 24 \\
\hline
\end{tabular}


Tabla 4. Parámetros de la regresión lineal (ln $\left.\mathrm{P}=\ln \mathrm{a}+\mathrm{b}^{*} \mathrm{LC}\right)$ según el modelo lineal simple para la relación entre la longitud corporal (LC) y peso seco (P) de algunos macroinvertebrados analizados. Abreviaturas en leyenda de Tabla 2.

Table 4. Parameters of the linear regression according to the simple linear model for the relationship between body length (LC) and dry weight (P) of some macroinvertebrates analyzed. Abbreviations in legend of Table 2.

\begin{tabular}{lcccccccc}
\hline Taxón & $\mathrm{a}$ & $\mathrm{b}$ & $\mathrm{R} 2$ & $\mathrm{XL}$ & $\mathrm{RL}$ & $\mathrm{XP}$ & $\mathrm{RP}$ & $\mathrm{n}$ \\
\hline $\begin{array}{l}\text { Ephemeroptera } \\
\text { C. penai }\end{array}$ & $0.09 \pm 0.02$ & $008 \pm 001$ & 0.84 & 3.42 & $1.2-6.4$ & 0.197 & $0.010-0.830$ & 32 \\
Coleoptera & & & & & & & & \\
Austrelmis sp. (L) & $-0.35 \pm 0.03$ & $0.15 \pm 0.01$ & 0.90 & 4.28 & $1.9-7.6$ & 0.2 .71 & $0.007-0.920$ & 40 \\
Cylloepus sp. (A) & $-0.71 \pm 0.43$ & $0.43 \pm 0.11$ & 0.39 & 3.93 & $3.5-4.4$ & 0.987 & $0.510-1.590$ & 25 \\
Microcylloepus sp. (A) & $-0.34 \pm 0.11$ & $0.27 \pm 0.05$ & 0.57 & 2.03 & $1.7-2.6$ & 0.204 & $0.080-0.420$ & 22 \\
\hline
\end{tabular}

En nueve grupos, el modelo exponencial fue el que mejor explico la relación entre las variables analizadas. Entre ellos las efémeras Leptohyphes eximius y Trichorythodes popayanicus, el coleóptero Helichus sp., el tricóptero Smicridea sp. y los quironómidos Tanypodinae, presentaron valores de $\mathrm{R}^{2}$ mayores a 0.90 (Tabla 3). También con este modelo se obtuvieron las correlaciones

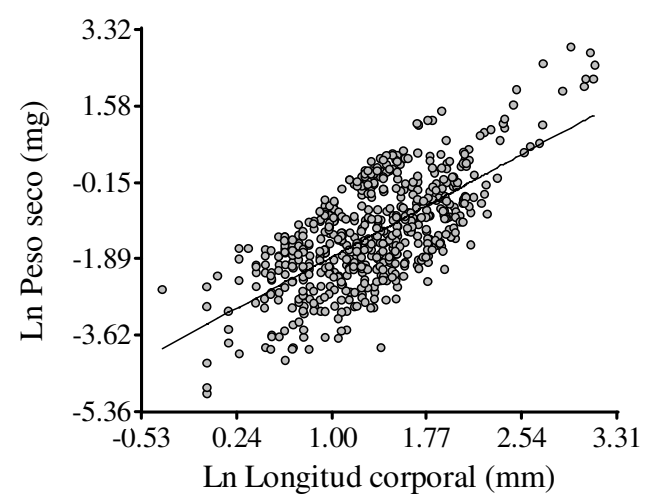

Figura 1. Relación entre la longitud corporal (transformada a ln) y el peso seco, según el modelo potencial, para los insectos bentónicos del arroyo Achiras.

Figure 1. Relationship between body length (ln transformed) and dry weight, according to the power model, for the benthic insects of Achiras stream. más bajas para adultos de Heterelmis sp. y Macrelmis sp. En los cuatro grupos restantes, que incluyen a Camelobaetidius penai, larvas de Austrelmis sp. y adultos de Cylloepus sp. y Microcylloepus sp., la regresión lineal simple ajustó mejor los valores de longitud corporal y peso (Tabla 4). En las efémeras y las larvas de Austrelmis sp. los $\mathrm{R}^{2}$ fueron superiores a 0.8 , pero en los coleópteros adultos la longitud corporal solo explico entre el 39\% y 57\% del peso.

Las ecuaciones predictivas para los niveles de familia y orden se calcularon utilizando los tres modelos antes descriptos. Las regresiones que mejor asociaron la relación entre P y LC para cada taxón se presentan en la Tabla 5. La relación entre las dos variables en las larvas de Austrelmis sp., Elmidae, Tanypodinae, Chironomidae y Simulium sp., y los adultos de Heterelmis sp., Macrelmis sp., Helichus sp. y Elmidae, resultaron igualmente explicadas por más de un modelo. (Tablas 2, 3, 4 y 5).

A partir de los registros de talla y peso de los insectos y de todos los macroinvertebrados medidos se estimaron dos ecuaciones generales que se ajustaron al modelo potencial. Para la clase Insecta (Figura 1) se obtuvo

Tabla 5. Ecuaciones estimadas a nivel de Familia y Orden, según el modelo potencial (1); exponencial (2) y lineal simple (3). Abreviaturas en leyenda de Tabla 2.

Table 5. Estimated equations for Family and Order levels, according to the power model (1), exponential model (2) and simple linear model (3). Abbreviations in legend of Table 2.

\begin{tabular}{|c|c|c|c|c|c|c|c|c|}
\hline Taxón & a ó $\ln$ a & $\mathrm{b}$ & $\mathrm{R}^{2}$ & XL & RL & $\mathrm{XP}$ & RP & $\mathrm{n}$ \\
\hline \multicolumn{9}{|l|}{ Ephemeroptera } \\
\hline Baetidae $^{(1)}$ & $-4.56 \pm 0.09$ & $2.17 \pm 0.07$ & 0.94 & 3.55 & $1.0-7.8$ & 0.211 & $0.007-1.200$ & 64 \\
\hline Leptohyphidae ${ }^{(2)}$ & $-2.91 \pm 0.06$ & $0.50 \pm 0.02$ & 0.93 & 3.22 & $0.7-6.6$ & 0.387 & $0.045-1.650$ & 65 \\
\hline Ephemeróptera (2) & $-3.31 \pm 0.11$ & $0.49 \pm 0.03$ & 0.7 & 3.39 & $0.7-7.8$ & 0.299 & $0.007-1.650$ & 139 \\
\hline \multicolumn{9}{|l|}{ Coleoptera } \\
\hline Elmidae (L) ${ }^{(1)}$ & $-5.06 \pm 0.19$ & $2.26 \pm 0.12$ & 0.8 & 4.8 & $1.9-10.5$ & 0.308 & $0.007-1.720$ & 88 \\
\hline Elmidae (L) ${ }^{(3)}$ & $-0.30 \pm 0.03$ & $0.12 \pm 0.01$ & 0.8 & 4.8 & $1.9-10.5$ & 0.308 & $0.007-0.720$ & 88 \\
\hline Elmidae $(\mathrm{A})^{(1)}$ & $-3.21 \pm 0.07$ & $2.33 \pm 0.06$ & 0.94 & 3.26 & $1.7-5.1$ & 0.723 & $0.080-1.740$ & 94 \\
\hline Elmidae (A) ${ }^{(3)}$ & $-0.69 \pm 0.04$ & $0.43 \pm 0.01$ & 0.94 & 3.26 & $1.7-5.1$ & 0.723 & $0.080-1.740$ & 94 \\
\hline \multicolumn{9}{|l|}{ Diptera } \\
\hline Chironomidae (L) ${ }^{(1)}$ & $-4.36 \pm 0.12$ & $1.62 \pm 0.07$ & 0.84 & 4.93 & $1.6-9.6$ & 0.196 & $0.020-0.810$ & 95 \\
\hline Chironomidae (L) ${ }^{(2)}$ & $-3.59 \pm 0.08$ & $0.33 \pm 0.02$ & 0.84 & 4.93 & $1.6-9.6$ & 0.196 & $0.020-0.810$ & 95 \\
\hline Diptera (L) ${ }^{(2)}$ & $-3.23 \pm 0.08$ & $0.29 \pm 0.02$ & 0.73 & 4.62 & $1.6-9.6$ & 0.194 & $0.020-0.810$ & 125 \\
\hline
\end{tabular}




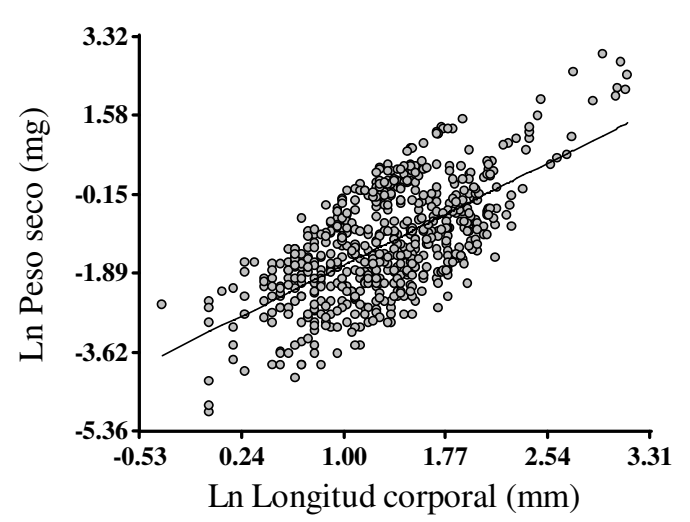

Figura 2. Relación entre la longitud corporal (transformada a ln) y el peso seco, según el modelo potencial, para todos los macroinvertebrados bentónicos del arroyo Achiras.

Figure 2. Relationship between body length (ln transformed) and dry weight, according to the power model, for all benthic macroinvertebrates of Achiras stream.

un valor de a igual a 0.03 y b igual a 1.52, resultando la expresión $\ln \mathrm{P}=-3.35+1.52 * \ln$ LC $\left(P<0.0001\right.$ y $\left.\mathrm{R}^{2}=0.47\right)$. Para la totalidad de los macroinvertebrados del arroyo Achiras los valores de a y b fueron 0.0015 y 1.46, respectivamente, y la ecuación de regresión fue $\ln P=-3.18+1.46^{*} \ln \mathrm{LC}\left(P<0.0001\right.$ y $\left.\mathrm{R}^{2}=0.42\right)$ (Figura 2).

\section{DisCUSIÓN}

En un análisis sobre estimaciones de biomasa de insectos acuáticos de distintos lugares geográficos Johnston \& Cunjak (1999) demostraron que las relaciones longitud-peso pueden producir predicciones muy diferentes, incluso para los taxones estrechamente relacionados. Estas diferencias pueden representar verdaderas variaciones espaciotemporal o taxonómica, o ser atribuidas a aspectos metodológicos relacionados con el tipo de conservantes de las muestras zoológicas o con los modelos de regresión aplicados (Johnston \& Cunjak 1999).

En individuos preservados, la biomasa es propensa a pérdidas que dependen del tiempo de preservación, el conservante y el grupo taxonómico (Howmiller 1972). Si bien en algunos estudios se utilizan especímenes frescos, en la mayoría se trabaja con material congelado o preservado químicamente. Para las regresiones estimadas en este trabajo se emplearon especímenes conservados en formol al $4 \%$, ya que este conservante puede proveer estimaciones de peso seco muy cercanas a aquellas en las que se utilizan animales frescos (Benke et al. 1999; Stoffels et al. 2003). No obstante, es aconsejable que cuando se empleen las ecuaciones predictivas para estimar la biomasa a partir de medidas de longitud se tenga especial cuidado en considerar que la validez de las mismas será mayor cuando más semejantes sean las condiciones de preservación de las muestras.

Uno de los principales referentes en estudios biométricos es el trabajo de Smock (1980), en el cual se propone que para estimar la biomasa de insectos acuáticos lo más apropiado es la ecuación potencial $\left(\mathrm{P}=\mathrm{a}^{*} \mathrm{X}^{\mathrm{b}}\right)$, y que el valor de $\mathrm{b}$ es cercano a tres y varía entre dos y cuatro. Sin embargo, otros autores observaron que en algunos casos son más convenientes otros modelos de regresión como el lineal simple o el exponencial (Meyer 1989; Burgeherr \& Meyer 1997; Cressa 1986; 1999a,b; Benke et al. 1999; González et al. 2002). En esta investigación, se emplearon los tres modelos mencionados y se determinó cual de ellos se ajusta mejor a cada uno de los taxones estudiados. En la mayoría de las predicciones a nivel de especie, género y subfamilia se obtuvieron regresiones muy significativas empleando el modelo potencial. Elmodelo exponencial explicó mejor la relación entre las variables en L. eximius, T. popayanicus, Helichus sp., Smicridea sp. y Tanypodinae. Para C. penai el modelo lineal permitió estimar la regresión con mayor significación que los otros dos modelos probados. Mientras que para las larvas de Austrelmis sp., tanto con la regresión lineal como con la potencial se obtuvo el mismo coeficiente de determinación. En coincidencia con Wenzel et al. (1990) consideramos que las disimilitudes entre los resultados obtenidos empleando diferentes modelos decrecerán si se utiliza un mayor número de especímenes. En efecto, si se consideran los valores de coeficiente de determinación obtenidos en función del número de individuos medidos, se observa que en todos los casos en que se trabajó con 30 ó más organismos, el $\mathrm{R}^{2}$ resultó siempre superior a 0.7. Mientras que cuando el número de individuos analizados fue inferior a 25 , los resultados fueron más variables (desde 0.99 a 0.2).

En las ecuaciones de regresión que relacionan talla y peso, el valor de la pendiente (b) representa el grado de incremento de peso seco por unidad de longitud. Para todos los grupos taxonómicos analizados en este trabajo, excepto para las larvas de Macrelmis sp., los valores de b fueron menores a tres, lo que coincide con los resultados obtenidos para el bentos de ríos venezolanos (Cressa 1999a) y arroyos brasileños (Becker et al. 2009). A su 
vez, dicho parámetro fue comparativamente menor que el estimado para invertebrados bentónicos de otros ríos argentinos (Miserendino 2001); y del hemisferio norte (Smock 1980; Burgherr \& Meyer 1997; Benke et al. 1999). Si el crecimiento es alométrico y puede explicarse según un modelo potencial, cuanto más cercano a tres sea el exponente b, más perfecta será la relación cúbica entre la longitud y el peso de los organismos (Benke et al. 1999). Cuando más se aleje b de este valor, mayor será la posibilidad de que la relación entre las variables de longitud y biomasa se ajuste a través de un modelo exponencial.

El coeficiente de determinación promedio hallado en nuestro estudio fue de 0.77, valor que se ubica dentro del rango de variación de los $\mathrm{R}^{2}$ presentados por otros autores (Smock 1980; Benke 1996; Burgherr \& Meyer 1997; Benke et al. 1999; Cressa 1999a,b; Miserendino 2001; González 2002). Estos valores están indicando que, para la fauna analizada, un elevado porcentaje del incremento del peso seco está explicado por el aumento de la longitud corporal. Sin embargo en los Elmidae adultos (excepto Austrelmis sp.) y en las pupas de Simulium sp. se obtuvieron coeficientes de determinación bajos con los tres modelos. Es probable que esto se deba a que tanto las pupas de simúlidos como los adultos de estos coleópteros presentan escasa variación de longitud corporal (rangos cercanos a $1 \mathrm{~mm}$ ), por lo cual individuos con tallas semejantes presentaron diferente peso y disminuye así el valor de los coeficientes en las regresiones estimadas. Futuros estudios con mayor número de especímenes y metodologías de medición más precisas permitirán ajustar estos resultados.

Entre los dípteros, para la familia Chironomidae, dos modelos se ajustaron adecuadamente: el potencial y el exponencial. Existen algunos antecedentes de relaciones potenciales de longitud-biomasa para esta familia (Meyer 1989; Smock 1980; Benke et al. 1999; Burgherr \& Meyer 1997). Estos estudios muestran valores de $b$ mucho mayores y una gran variabilidad en los parámetros de los modelos ajustados (Stoffels et al. 2003). También con el primero de estos modelos, Smock (1980) estimó para la familia Simuliidae un valor de b más elevado. Al considerar todos los registros de larvas de Diptera, la ecuación que mejor explicó la relación fue la exponencial, con una baja pendiente. Las larvas de dípteros tienen cuerpos muy elongados, tubulares y relativamente lisos, lo que determina poca superficie corporal por unidad de longitud. La forma y la relativamente pequeña cantidad de áreas quitinizadas explican el bajo incremento de peso por unidad de longitud (Cressa 1999a).

Los coeficientes de determinación de las ecuaciones ajustadas para todos los insectos acuáticos y todos los macroinvertebrados del arroyo Achiras fueron bajos (menores a $50 \%$ ). Como fue propuesto por Smock (1980), Miserendino (2001) y Sabo et al. (2002), los modelos de regresión aplicados a grupos con una resolución taxonómica mayor explican una mayor proporción de la variación del peso seco respecto de las regresiones desarrolladas al nivel taxonómico de orden. Por esta razón, cuando se necesita precisión, se aconseja elegir las ecuaciones predictivas basadas en el menor nivel taxonómico posible. No obstante, los modelos de regresión a nivel de grandes grupos se utilizan a menudo cuando por cuestiones de tiempo o presupuesto se evita el esfuerzo de identificaciones más precisas.

Para todos los taxa analizados en este estudio pudo ser calculado, con mayor o menor grado de ajuste, algún modelo de regresión. Sin embargo, de la comparación con los estudios realizados por otros autores se observa una gran variabilidad en los parámetros de los modelos ajustados (Meyer 1989; Smock 1980; Benke et al. 1999; Stoffels et al. 2003). Estas discrepancias pueden deberse a que en cada estudio se analizan distintos grupos faunísticos, con metodologías de preservación y medición que no siempre fueron idénticas. Por otra parte, se debe tener en cuenta que las variaciones de biomasa durante el crecimiento de los organismos serán diferentes según las condiciones tróficas o genéticas, y las características bióticas y fisicoquímicas del ambiente en que se desarrollan. Por este motivo se sugiere que para determinar la biomasa a partir de ecuaciones matemáticas ya existentes, se empleen estimaciones calculadas para la fauna local o regional.

Agradecimientos: A la Secretaría de Ciencia y Técnica de la Universidad Nacional de Río Cuarto por el soporte financiero y a los dos revisores anónimos que contribuyeron a mejorar este trabajo.

\section{BiBLIOGRAFÍA}

Armitage, PD. 2006. Long-term faunal changes in a regulated and an unregulated stream-Cow Green thirty years on. River Research and Applications, 22:947-966.

BENKE, AC. 1996. Secondary production of macroinvertebrates. Pp. 557-578 en: Hauer, FR \& GA Lamberti (eds.). Methods in stream ecology. Academic 
Press, San Diego, CA.

Benke, AC; AD Huryn; la Smock \& JB Wallace. 1999. Length-mass relationships for freshwater macroinvertebrates in North America with particular reference to the southeastern United States. J. N. Am. Benthol. Soc., 18:308-343.

BeCKer, B; MS Moretti \& M Callisto. 2009. Length-dry mass relationships for a typical shredder in Brazilian streams (Trichoptera: Calamoceratidae) Aquatic Insects, 31:227-234.

Burgherr, P \& EI Meyer. 1997. Regression analysis of linear body dimensions vs. dry mass in stream macroinvertebrates. Arch. Hydrobiol., 139:101-112.

CRESSA, C. 1986. Estimaciones de peso seco en funcion de la longitud cefalica y clases de tamaño en Campsurus sp. (Ephemeroptera, Polymitarcidae). Acta Cientifica Venezolana, 37:170-173.

CRessa, C. 1999a. Dry mass estimates of some tropical aquatic insects. Rev. Biol. Trop., 47:133-141.

CRESSA, C. 1999b. Dry mass estimation of tropical aquatic insects using different short-term preservation methods. Rev. Biol. Trop., 47:143-149.

Di Rienzo, JA; F Casanoves; MG Balzarini; L GonZÁlez; M TABLADA; ET AL. 2010. InfoStat, software Estadística, v. 2011. Grupo InfoStat, Facultad de Ciencias Agrarias, Universidad Nacional de Córdoba. Argentina.

Domínguez, E \& HR Fernández (eds.). 2009. Macroinvertebrados bentónicos sudamericanos: Sistemática y biología. Fundación M. Lillo. Tucumán, Argentina. Pp. 654.

FERNÁNDEZ, HR \& E Domínguez (eds.). 2001. Guía para la determinación de los artrópodos bentónicos sudamericanos. EUDET. Tucumán, Argentina. Pp. 282.

GonzÁlez, JM; A Basaguren \& J Pozo. 2002. Size-mass relationships of stream invertebrates in a northern Spain stream. Hydrobiologia, 489:131-137.

GuAlDONI, CM \& AM OBerto. 2012. Estructura de la comunidad de macroinvertebrados del arroyo Achiras (Córdoba, Argentina): análisis previo a la construcción de una presa. Iheringia, 102:177-186.

HOWMILLER, RP. 1972. Effect of preservatives on weights of some common macrobenthic invertebrates. Trans. Am. Fish Soc., 101:743-746.

Johnston, AT \& RA Cynjak. 1999. Dry mass-length relationships for benthic insects: a review with new data from Catamaran Brook, New Brunswick, Canada. Freshwater Biology, 41:653-674

Lopreto, EC \& G Tell. 1995. Ecosistemas de aguas continentales, Metodología para su estudio. Tomo III. Ediciones Sur, La Plata, Argentina. Pp. 1401.

MeYer, E. 1989. The relationship between body length parameters and dry mass in running water invertebrates. Arch. Hydrobiol., 117:191-203.

MiserendinO, ML. 2001 Length-mass relationships for macroinvertebrates in freshwater environments in Patagonia (Argentina). Ecol. Austral, 11:3-8.

Poff, NL \& JKH Zimmerman. 2010. Ecological responses to altered flow regimes: a literature review to inform environmental flows science and management. Freshwater Biology, 55:194-20.

SABO, JL; JL BAstow \& ME Power. 2002. Lengthmass relationships for adult aquatic and terrestrial invertebrates in a California watershed. J. N. Am. Benthol. Soc., 21:336-343

SMOCK, LA. 1980. Relationships between body size and biomass of aquatic insects. Freshwat. Biol., 10:375-383.

Stoffels, RJ; S Karbe \& RA PATERSON. 2003. Length-mass models for some common New Zealand littoral-benthic macroinvertebrates, with a note on within-taxon variability in parameter values among published models. New Zeal J. Mar. Fresh., 37:449-460.

TOWERS, DJ; IMHENDERSON \& CJ VeltMAn. 1994. Predicting dry weight of New Zealand aquatic macroinvertebrates from linear dimensions. New Zeal J. Mar. Fresh., 28:159-166.

Wenzel, F; E Meyer \& J SChWOerbel. 1990. Morphometry and biomass determination of dominant mayfly larvae (Ephemeroptera) in running waters. Arch. Hydrobiol., 118:31-46. 